\title{
Co-administration of vancomycin and piperacillin-tazobactam is associated with increased renal dysfunction in adult and pediatric burn patients
}

Gabriel Hundeshagen ${ }^{1,2,3^{*}}$ D, David N. Herndon ${ }^{1,2}$, Karel D. Capek ${ }^{1,2}$, Ludwik K. Branski ${ }^{1,2,4}$, Charles D. Voigt ${ }^{1,2}$, Elizabeth A. Killion ${ }^{4}$, Janos Cambiaso-Daniel ${ }^{5}$, Michaela Sljivich ${ }^{1,2}$, Andrew De Crescenzo ${ }^{1,2}$, Ronald P. Mlcak ${ }^{1,2}$, Michael P. Kinsky ${ }^{6}$, Celeste C. Finnerty ${ }^{1,2}$ and William B. Norbury ${ }^{1,2}$

\begin{abstract}
Background: Burn patients are prone to infections which often necessitate broad antibiotic coverage. Vancomycin is a common antibiotic after burn injury and is administered alone $(V)$, or in combination with imipenem-cilastin (V/IC) or piperacillin-tazobactam (V/PT). Sparse reports indicate that the combination V/PT is associated with increased renal dysfunction. The purpose of this study was to evaluate the short-term impact of the three antibiotic administration types on renal dysfunction.

Methods: All pediatric and adult patients admitted to our centers between 2004 and 2016 with a burn injury were included in this retrospective review if they met the criteria of exposition to either $\mathrm{V}, \mathrm{V} / \mathrm{IC}$, or V/PT for at least $48 \mathrm{~h}$, had normal baseline creatinine, and no pre-existing renal dysfunction. Creatinine was monitored for 7 days after initial exposure; the absolute and relative increase was calculated, and patient renal outcomes were classified according to the Kidney Disease Improving Global Outcomes (KDIGO) criteria depending on creatinine increases and estimated creatinine clearance. Secondary endpoints (demographic and clinical data, incidences of septicemia, and renal replacement therapy) were analyzed. Antibiotic doses were modeled in logistic and linear multivariable regression models to predict categorical KDIGO events and relative creatinine increase.
\end{abstract}

Results: Out of 1449 patients who were screened, 718 met the inclusion criteria, 246 were adults, and 472 were children. Between the study cohorts V, V/IC, and V/PT, patient characteristics at admission were comparable. V/PT administration was associated with a statistically higher serum creatinine, and lower creatinine clearance compared to patients receiving $V$ alone or $V / I C$ in adults and children after burn injury. The incidence of KDIGO stages 1,2 , and 3 was higher after V/PT treatment. In children, the incidence of KDIGO stage 3 following administration of V/PT was greater than after V/IC. In adults, the incidence of renal replacement therapy was higher after V/PT compared with $\mathrm{V}$ or V/IC. Multivariate modeling demonstrated that V/PT is an independent predictor of renal dysfunction.

Conclusion: Co-administration of vancomycin and piperacillin-tazobactam is associated with increased renal dysfunction in pediatric and adult burn patients when compared to vancomycin alone or vancomycin plus imipenem-cilastin. The mechanism of this increased nephrotoxicity remains elusive and warrants further scientific evaluation.

\footnotetext{
* Correspondence: gabrielhundeshagen@gmail.com

${ }^{1}$ Department of Surgery, University of Texas Medical Branch, 301 University Blvd., Galveston, TX 77555, USA

${ }^{2}$ Shriners Hospitals for Children, 815 Market St., Galveston, TX 77550, USA

Full list of author information is available at the end of the article
} 


\section{Background}

Renal failure is a common complication in critically ill patients, particularly in burn patients [1-3]. Acute kidney injury (AKI) that is sustained alongside thermal injury significantly worsens morbidity and mortality in pediatric and adult patients $[4,5]$. Over recent decades, the prevalence of multidrug-resistant organisms has been steadily increasing [6, 7], limiting antibiotic treatment options for affected patients and necessitating greater use of aggressive therapeutic antimicrobial combinations [8]. Burn patients are particularly susceptible to infection with multidrug-resistant organisms owing to substantial loss of skin barrier function, wound contamination, nosocomial exposure to pathogens, and impaired postburn immune function [9-12]. Bacterial infection is a leading cause of death among burn patients [13] and is treated with various combinations of antimicrobials. Vancomycin (V) is a glycopeptide that inhibits cell wall synthesis in gram-positive bacteria; its spectrum of effectivity includes methicillin-resistant Staphylococcus aureus [14]. Nephrotoxicity is a side effect of intravenous vancomycin therapy [15]. Imipenem combined with its co-effector cilastin (IC) is a broad-spectrum carbapenem that is commonly used to treat burn-related local and systemic infection caused by Pseudomonas aeruginosa, Klebsiella, or Acinetobacter species [16]. Piperacillin, an extended-spectrum penicillin, combined with the $\beta$-lactamase tazobactam (PT) has activity against various gram-positive and gram-negative organisms, including Pseudomonas and Enterobacteria [17]. Imipenemcilastin and piperacillin-tazobactam share a similar profile of broad coverage, which enables a degree of interchangeability in burn care [18-20]. Recently, other groups have reported that the combination of vancomycin and piperacillin-tazobactam induces greater renal damage in non-critically ill patients than vancomycin alone [21] or the combination of vancomycin and cefepime [22]. Here, AKI associated with exposure to vancomycin alone $(\mathrm{V})$, vancomycin plus imipenem-cilastin (V/IC), or vancomycin plus piperacillin-tazobactam $(\mathrm{V} / \mathrm{PT})$ was quantified based on Kidney Disease Improving Global Outcomes (KDIGO) criteria [23] to determine whether any of these treatment combinations is indicated to be favored regarding nephrotoxicity in adult and pediatric burn patients.

\section{Methods}

\section{Patients and study design}

This study was approved by the Institutional Review Board of the University of Texas Medical Branch (UTMB). As per the Institutional Review Board, this retrospective chart review of de-identified patient data was exempt from additional consenting procedures. A cohort study was designed based on a retrospective chart review between the years 2004 and 2016. All consecutive patients admitted to the UTMB adult burn unit and Shriners Hospitals for Children -Galveston pediatric burn unit were included in the study if they met the following criteria: admitted for acute burn injury of any size; normal ageand sex-adjusted creatinine at admission; and receipt of either vancomycin alone $(\mathrm{V})$, vancomycin plus imipenemcilastin (V/IC), or vancomycin plus piperacillin-tazobactam (V/PT) intravenously for at least $48 \mathrm{~h}$ of therapeutic treatment. Exclusion criteria were: death upon admission or within the first $48 \mathrm{~h}$ following admission; admission for diagnosis other than burn injury; abnormal baseline creatinine; pre-injury renal failure or dialysis; crossing over between the study groups after first exposure to $\mathrm{V}, \mathrm{V} / \mathrm{IC}$, or V/PT; and incomplete demographic data. Upon admission, burn size and severity were recorded on Lund and Browder charts by the attending surgeon, and demographic data were recorded. If necessary, adult patients were resuscitated as guided by the Parkland formula as previously published $[24,25]$. Children were resuscitated according to the standardized Galveston formula as previously published [26, 27]: $5000 \mathrm{ml} / \mathrm{m}^{2}$ total body surface area (TBSA) burned $+2000 \mathrm{ml} / \mathrm{m}^{2}$ TBSA lactated Ringer's solution administered in increments over the first $24 \mathrm{~h}$ after admission. All patients with full-thickness burns were treated surgically with complete burn wound excision and grafting with autoor homograft, depending on skin graft availability [26, 27]. In adults, $\mathrm{V}$ was administered identically in all study cohorts at 1000-1500 mg every $12 \mathrm{~h}$, and the dose adjusted daily to maintain a trough of $10-15 \mu \mathrm{g} / \mathrm{ml}$. In children, the starting dose of $\mathrm{V}$ was $15 \mathrm{mg} / \mathrm{kg}$ every $6 \mathrm{~h}$ and the dose was adjusted daily to maintain a trough of $10-15 \mu \mathrm{g} / \mathrm{ml}$. In adults, 500-1000 mg IC was administered every 6-8 h, with a maximum daily dose of $50 \mathrm{mg} / \mathrm{kg}$ or $4 \mathrm{~g}$, whichever was lower. In children, $15-25 \mathrm{mg} / \mathrm{kg}$ IC was given every $6 \mathrm{~h}$ with daily maximum doses of 2-4 g depending on infection severity. In adults and adolescents $>40 \mathrm{~kg}, 375-4500 \mathrm{mg}$ PT was administered every 6-8 hours with a maximum $18 \mathrm{~g}$ per day; children $>$ 9 months and $<40 \mathrm{~kg}$ received $100 \mathrm{mg} / \mathrm{kg}$ PT every $6 \mathrm{~h}$.

\section{Cohorts and endpoints}

Patients were allocated to one of the three cohorts based on their exposure to $\mathrm{V}, \mathrm{V} / \mathrm{IC}$, or $\mathrm{V} / \mathrm{PT}$ as defined above. Administered doses of antibiotics were recorded and adjusted to patient body weight as the average daily dose (D; $\mathrm{mg} / \mathrm{kg} /$ day).

For up to 7 days after exposure, serum creatinine $(\mathrm{Cr})$ concentration was monitored daily (a daily average if more than one value per day). The following were determined: baseline concentration $\left(\mathrm{Cr}_{\mathrm{BL}} ; \mathrm{mg} / \mathrm{dl}\right)$; maximum concentration $\left(\mathrm{Cr}_{\max }=\right.$ maximum value recorded during 7 days post exposition; $\mathrm{mg} / \mathrm{dl}$ ); absolute increase 
$\left(\mathrm{Cr} \Delta_{\mathrm{a}}=\mathrm{Cr}_{\max }-\mathrm{Cr}_{\mathrm{BL}} ; \mathrm{mg} / \mathrm{dl}\right) ;$ and relative increase $\left(\mathrm{Cr} \Delta_{\%}=\left(\mathrm{Cr}_{\max }-\mathrm{Cr}_{\mathrm{BL}}\right) / \mathrm{Cr}_{\mathrm{BL}} \times 100\right)$.

For the study period, patient status was classified according to the creatinine-based KDIGO stages of acute kidney injury [28]: 1 ( $\geq 1.5$-fold serum creatinine increase, increase by $\geq 0.3 \mathrm{mg} / \mathrm{dL}$ ), 2 ( $\geq 2$-fold serum creatinine increase), and 3 ( $\geq 3$-fold serum creatinine increase or $\geq 4 \mathrm{mg} / \mathrm{dl}$ ).

For 7 days after exposure, daily creatinine clearance was estimated $(\mathrm{eCrCl})$ according to $\mathrm{Cr}$-based formulas. For adult patients, Cockcroft Gault's formula was used [29]: $\mathrm{eCrCl}=(140-$ age $($ years $)) \times$ bodyweight $(\mathrm{kg}) \times 0.85$ (if female) $/ 72 \times \mathrm{Cr}(\mathrm{mg} / \mathrm{dL})$. For children, the Léger formula was used [30]: eCRCL $=(0.641 \times$ weight $(\mathrm{kg})) / \mathrm{Cr}$ $(\mathrm{mg} / \mathrm{dL})+\left(0.00131 \times\right.$ height $\left.^{2}\left(\mathrm{~cm}^{2}\right)\right) / \mathrm{Cr}(\mathrm{mg} / \mathrm{dL})$.

The following were determined: baseline $\mathrm{eCrCl}\left(\mathrm{eCrCl}_{\mathrm{BL}}\right.$; $\mathrm{mg} / \mathrm{dl})$; minimum eCrCl $\left(\mathrm{eCrCl}_{\min }=\right.$ minimum value recorded during 7 days post exposition; $\mathrm{mg} / \mathrm{dl}$ ); absolute decrease $\left(\mathrm{eCrCl} \Delta_{\mathrm{a}}=\mathrm{eCrCl}_{\text {min }}-\mathrm{eCrCl}_{\mathrm{BL}} ; \mathrm{mg} / \mathrm{dl}\right)$; and relative decrease $\left(\mathrm{eCrCl} \Delta_{\%}=\left(\mathrm{eCrCr}_{\min }-\mathrm{eCrCl}_{\mathrm{BL}}\right) / \mathrm{eCrCl} \mathrm{BL}_{\mathrm{BL}} \times 100\right)$.

Demographic data, concomitant inhalation injury, and septicemia, as well as occurrence of dialysis throughout hospitalization, were noted.

\section{Statistical analysis}

Continuous, normally distributed data are presented as mean \pm standard deviation and were compared using oneway analysis of variance (ANOVA). Nonparametric continuous data were analyzed using the Kruskal-Wallis test. Dichotomous and categorical variables were analyzed using the chi-squared test. A multiple general linear regression model was fit to the continuous response $\left(\mathrm{Cr} \Delta_{\%}\right)$, with average daily dose of $\mathrm{V}, \mathrm{IC}$, and $\mathrm{PT}$ as independent variables, while adjusting for TBSA percentage burned, age and the presence of inhalation injury. Logistic regression analyses for KDIGO stages 1, 2, and 3 were performed based on antibiotic doses as independent variables. We performed stepwise logistic regression modeling while selecting variables based on minimization of Akaike Information Criterion [31]. Goodness of fit of the logistic regression models was confirmed by the HosmerLemeshow test [32]. Due to the low incidence of AKI events, adult and pediatric patients were analyzed jointly in linear and logistic regression models. As indicated, predictors and responses were transformed for better fitting of model assumptions and the independence of variables confirmed by correlation coefficients. In all comparisons, statistical significance was accepted at $p<0.05$.

\section{Results}

\section{Patient characteristics}

During the study period, 1449 patients were admitted (739 adults and 710 pediatric patients) to both centers. Figure 1 illustrates the inclusion process into the analysis. Data from 718 (246 adults and 472 children) patients were evaluated. The median sampling time point was the year 2014 for $\mathrm{V}$ and V/IC, and 2011 for V/PT.

Characteristics of adult and pediatric patients are shown in Table 1 . The study cohorts were comparable with regard to age, sex, TBSA burned, $\mathrm{CR}_{\mathrm{BL}}$, and incidence of concomitant inhalation injury for both children and adults.

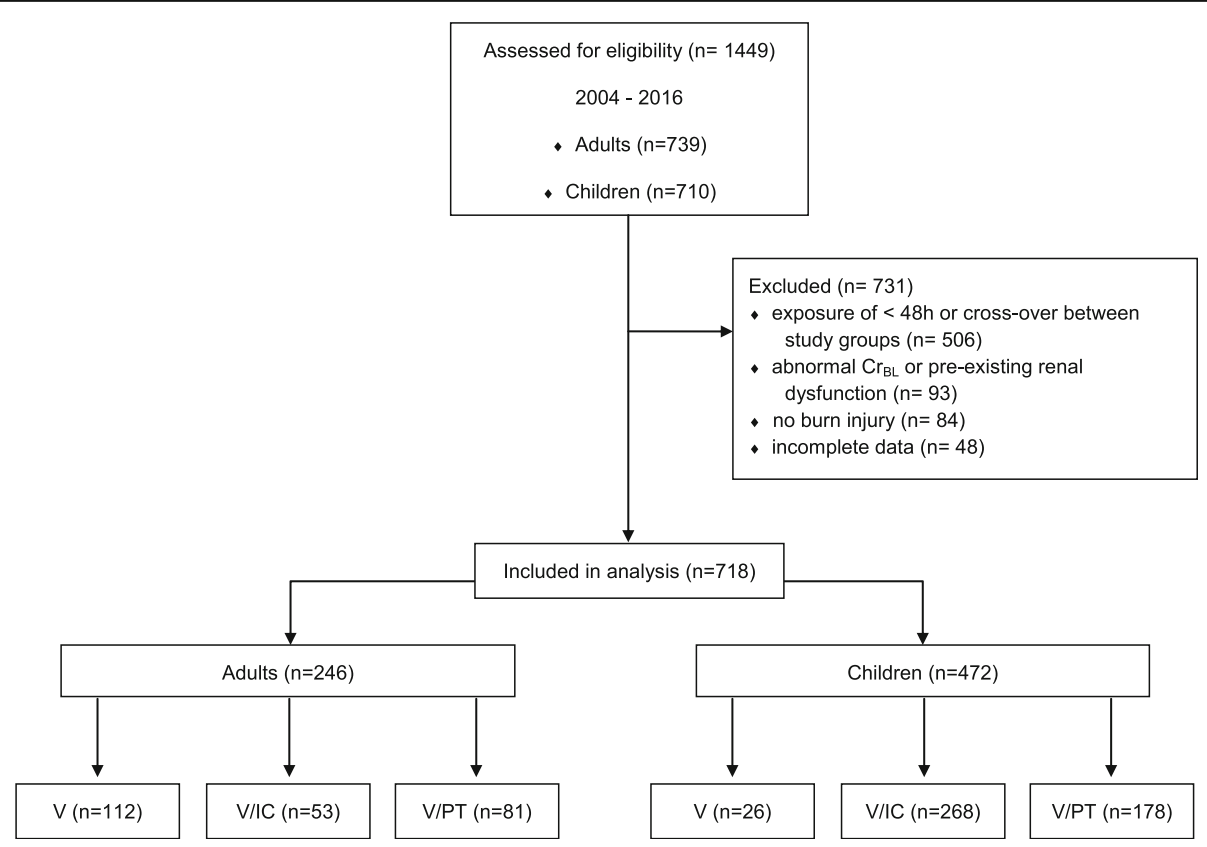

Fig. 1 Patient enrollment. $C r_{B L}$ baseline creatinine concentration, $V$ vancomycin, $V / I C$ vancomycin plus imipenem-cilastin, V/PT vancomycin plus piperacillin-tazobactam 
Table 1 Patient characteristics at admission

\begin{tabular}{|c|c|c|c|c|}
\hline & V & $\mathrm{V} / \mathrm{IC}$ & V/PT & $p$ value \\
\hline Adults & $n=112$ & $n=53$ & $n=81$ & \\
\hline Age (years) & $46 \pm 16$ & $44 \pm 16$ & $41 \pm 17$ & 0.10 \\
\hline Sex (male:female), $n$ & $70: 39$ & $41: 12$ & $78: 23$ & 0.09 \\
\hline TBSA (\%) & $13.5 \pm 15$ & $12 \pm 12$ & $15 \pm 17$ & 0.46 \\
\hline Inhalation injury, n (\%) & $11(10)$ & $6(11)$ & $10(10)$ & 0.90 \\
\hline Cause of burn, $n(\%)$ & & & & ns \\
\hline Flame & $94(84)$ & $68(90)$ & $87(88)$ & \\
\hline Scald & $7(6)$ & $2(2)$ & $6(6)$ & \\
\hline Electrical & $6(5)$ & $5(6)$ & $2(2)$ & \\
\hline Other & $5(5)$ & $1(2)$ & $4(4)$ & \\
\hline $\mathrm{CR}_{\mathrm{BL}}(\mathrm{mg} / \mathrm{dl})$ & $0.80 \pm 0.18$ & $0.79 \pm 0.19$ & $0.81 \pm 0.19$ & 0.96 \\
\hline Children & $n=26$ & $n=268$ & $n=178$ & \\
\hline Age (years) & $8 \pm 6$ & $7 \pm 5$ & $7 \pm 6$ & 0.62 \\
\hline Sex (male:female), $n$ & $17: 9$ & 165:103 & $114: 64$ & 0.83 \\
\hline TBSA (\%) & $32.0 \pm 18$ & $39 \pm 19$ & $40 \pm 18$ & 0.10 \\
\hline Inhalation injury, n (\%) & $3(12)$ & $48(18)$ & $19(11)$ & 0.10 \\
\hline Cause of burn, $n(\%)$ & & & & ns \\
\hline Flame & $13(50)$ & $137(51)$ & $105(59)$ & \\
\hline Scald & $7(26)$ & $102(38)$ & $57(32)$ & \\
\hline Electrical & $2(8)$ & $24(9)$ & $11(6)$ & \\
\hline Other & $4(15)$ & $5(2)$ & $5(2)$ & \\
\hline $\mathrm{CR}_{\mathrm{BL}}(\mathrm{mg} / \mathrm{dl})$ & $0.46 \pm 0.28$ & $0.50 \pm 0.22$ & $0.50 \pm 0.21$ & 0.70 \\
\hline
\end{tabular}

Values are shown as mean \pm SD unless otherwise indicated

$C R_{B L}$ baseline creatinine concentration, $I C$ imipenem-cilastin, $n s$ not significant,

PT piperacillin-tazobactam, TBSA total body surface area burned, $V$ vancomycin

\section{Antibiotic treatment}

Study subjects were exposed to antibiotic treatment at comparable times during the course of hospitalization; 93\% of $\mathrm{V}, 98 \%$ of $\mathrm{V} / \mathrm{IC}$, and $97 \%$ of $\mathrm{V} / \mathrm{PT}$ patients were exposed during the first $24 \mathrm{~h}$ after admission $(p=0.45)$. Average daily doses of vancomycin were comparable between cohorts for both children and adults, regardless of administration with IC, PT, or alone. The distribution of days of exposure (2-5 days) was comparable between treatment cohorts for both adults and children, with $>90 \%$ of pediatric subjects and $>65 \%$ of adults being exposed for 4 or more days. Based on the administration schedules described above, children received lower absolute doses of $\mathrm{V}$, $\mathrm{IC}$, and PT than adults $(p<0.0001)$ but higher average daily doses of V, IC, and PT per body weight (V: $50 \pm 17$ $\mathrm{mg} / \mathrm{kg} /$ day; IC: $55 \pm 21 \mathrm{mg} / \mathrm{kg} /$ day; PT: $257 \pm 62 \mathrm{mg} / \mathrm{kg} /$ day) than adult patients (V: $21 \pm 6 \mathrm{mg} / \mathrm{kg} /$ day; IC: $20 \pm 6$ $\mathrm{mg} / \mathrm{kg} /$ day; PT: $135 \pm 41 \mathrm{mg} / \mathrm{kg} /$ day $)(p<0.0001)$.

\section{Serum creatinine by treatment cohort Adults}

During 7 days after exposure to one of the treatments, the average absolute creatinine increase was greater in the V/PT group than in the $\mathrm{V}$ or $\mathrm{V} / \mathrm{IC}$ groups (V/PT: $0.26 \pm 0.62 \mathrm{mg} / \mathrm{dl} ; \mathrm{V}: 0.05 \pm 0.10 \mathrm{mg} / \mathrm{dl} ; \mathrm{V} / \mathrm{IC}: 0.06 \pm 0.09$ $\mathrm{mg} / \mathrm{dl} ; p<0.001$ for $\mathrm{V} / \mathrm{PT}$ vs. $\mathrm{V}$ and $p<0.01$ for $\mathrm{V} / \mathrm{PT}$ vs. V/IC; Fig. 2a). No difference was detected between $V$ and $\mathrm{V} / \mathrm{IC}$. The average relative increase in creatinine was greater in the $\mathrm{V} / \mathrm{PT}$ group than in the $\mathrm{V}$ or $\mathrm{V} / \mathrm{IC}$ groups (V/PT: $37 \pm 91 \%$; V: $8 \pm 20 \%$; V/IC: $7 \pm 11 \%$; $p<0.001$ for $\mathrm{V} / \mathrm{PT}$ vs. $\mathrm{V}$ and $p<0.01$ for V/PT vs. V/IC; Fig. $2 \mathrm{~b})$.

Absolute eCrCl decreased more after exposure to $\mathrm{V} /$ PT than to V (V/PT: $-26 \pm 39 \mathrm{ml} / \mathrm{min}$; V: $-10 \pm 28 \mathrm{ml} /$ min; $p<0.001$; Fig. 3a). Relative eCrCl decreased further in the $\mathrm{V} / \mathrm{PT}$ group than in the $\mathrm{V}$ or $\mathrm{V} / \mathrm{IC}$ groups $(\mathrm{V} / \mathrm{PT}$ : $17 \pm 21 \%$; V/IC: $-7 \pm 8 \%$; : $6 \pm 10 \% ; p<0.001$; Fig. $3 \mathrm{~b})$.

The rate of occurrence of KDIGO stage 1 was higher in the V/PT group (15\%) than in the $\mathrm{V}(2 \%)$ or $\mathrm{V} / \mathrm{IC}$ group $(0 \%)(p<0.001)$. KDIGO stage 2 was measured more frequently in the V/PT group $(7 \%)$ than in the $\mathrm{V}$ $(2 \%)$ or $\mathrm{V} / \mathrm{IC}$ group $(0 \%)(p<0.05)$. KDIGO stage 3 of AKI occurred more frequently after exposure to V/PT (5\%) compared to V (0\%) or V/IC (0\%) $(p<0.05$; Fig. 4a). The adjusted odds ratio of AKI stage 1 for V/PT vs. V/ IC was 18.4 (confidence interval 1.06-319.2).

\section{Children}

Over 7 days of exposure to one of the treatments, the average absolute creatinine increase was greater in the $\mathrm{V} / \mathrm{PT}$ group than in the $\mathrm{V} / \mathrm{IC}$ group (V/PT: $0.14 \pm 0.49$ $\mathrm{mg} / \mathrm{dl} ; \mathrm{V} / \mathrm{IC}: 0.05 \pm 0.08 \mathrm{mg} / \mathrm{dl} ; p<0.05$; Fig. $2 \mathrm{c})$. No differences were detected between $\mathrm{V}(\mathrm{V}: 0.05 \pm 0.07)$ and $\mathrm{V} / \mathrm{IC}$ or $\mathrm{V}$ and $\mathrm{V} / \mathrm{PT}$. The average relative increase in creatinine was greater in the $\mathrm{V} / \mathrm{PT}$ group than in the $\mathrm{V} /$ IC group (V/PT: $26 \pm 62 \%$; V/IC: $14 \pm 23 \% ; p<0.05$; Fig. 2d). No differences were found between $\mathrm{V}$ and $\mathrm{V} / \mathrm{IC}$ or $\mathrm{V}$ and $\mathrm{V} / \mathrm{PT}(\mathrm{V}: 16 \pm 21 \%)$.

No differences in absolute decrease of $\mathrm{eCrCl}$ were detected between the groups (Fig. 3c). Relative eCrCl decreased further in the V/PT group than in the $\mathrm{V} / \mathrm{IC}$ group (V/PT: $-18 \pm 2 \%$; V/IC: $-14 \pm 18 \%$; $p<0.05$; Fig. $3 d$ ).

The occurrence of KDIGO stage 3 of AKI was higher in the $\mathrm{V} / \mathrm{PT}$ group $(2 \%)$ than in the $\mathrm{V}(0 \%)$ or $\mathrm{V} / \mathrm{IC}$ group $(0 \%)(p<0.05$; Fig. $4 \mathrm{~b})$. The rate of occurrence of KDIGO stages 1 (V: 4\%; V/IC: 9\%; V/PT: 13\%) or 2 (V: 0\%; V/IC: $1 \%$; V/PT: $5 \%$ ) did not differ between the groups. The odds ratio of AKI stage 3 for V/PT vs. V/IC was 4.01 (confidence interval 1.05-15.4).

\section{Secondary endpoints}

As summarized in Additional file 1: Table S1, length of hospitalization, as well as the incidence of septicemia and mortality, were comparable between the treatment cohorts both in adults and children. However, the incidence of hemodialysis during the course of acute hospitalization was significantly greater in adults treated with V/PT (3\%) than in those treated with $\mathrm{V}$ or $\mathrm{V} / \mathrm{IC}$ (both $0 \%, p=0.03$ ). 

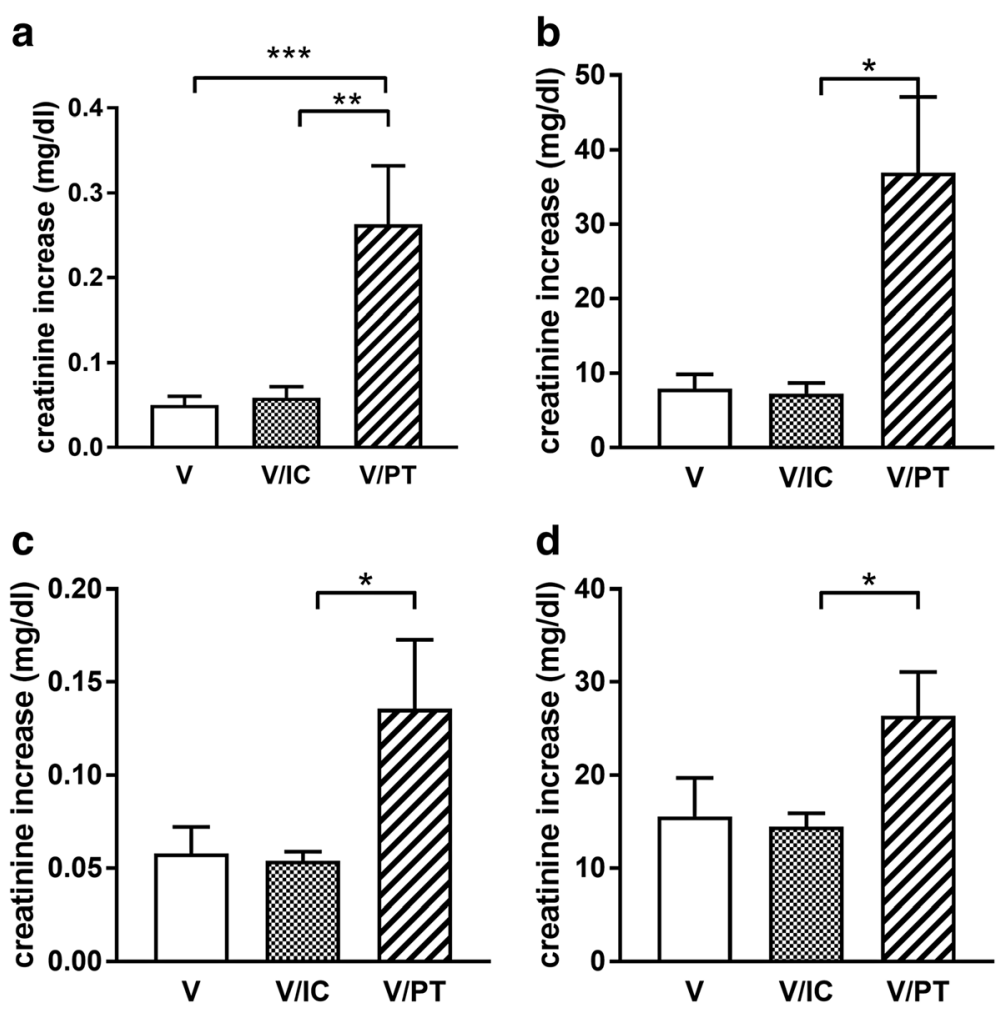

Fig. 2 Adult average (a) absolute $(\mathrm{mg} / \mathrm{dl})$ and $(\mathbf{b})$ relative (\%) creatinine increase 7 days following exposure to $V, V / I C$, and V/PT. Child average (c) absolute $(\mathrm{mg} / \mathrm{dl})$ and $(\mathbf{d})$ relative (\%) creatinine increase 7 days following exposure to $\mathrm{V}, \mathrm{V} / \mathrm{IC}$, and V/PT. ${ }^{*} p<0.05,{ }^{* *} p<0.01,{ }^{* * *} p<0.001$. IC imipenem-cilastin, PT piperacillin-tazobactam, $V$ vancomycin

There was no difference between the pediatric study cohorts regarding the use of renal replacement therapy (RRT). Clinical data of patients who received RRT are summarized in Table 2.

\section{Statistical modeling}

Multivariate models are summarized in Tables 3 and 4. In a joint linear model for the entire patient cohort, while controlling for TBSA, age, and the presence of inhalation injury, the dose of PT had a significant linear effect on $\mathrm{Cr} \Delta_{\%}(p<0.001)$. In logistic regression models of all patients combined, after stepwise elimination of covariates, the dose of PT predicted KDIGO stage 1 $(p=0.03), 2(p=0.004)$, and $3(p=0.009)$. There were no significant dose-dependencies for IC or V.

\section{Discussion}

We demonstrate in a large patient cohort that adult and pediatric burn patients sustain independent short-term renal effects of antibiotic treatment with vancomycin plus piperacillin-tazobactam which are not present in patients treated with combinations of imipenem-cilastin and vancomycin or vancomycin alone.

These data are consistent with several smaller reports and case series; studies in diabetic adults with osteomyelitis
[33] and heterogeneous non-critically ill patient populations hypothesized that the combination of $\mathrm{V} / \mathrm{PT}$ induces increased incidences of nephrotoxicity [21, 34]. What is unique about our study design is that we provide a large body of pediatric data and provide a study design that allows for detection of direct effects of drug exposition on selective biomarkers and short-term clinical outcomes. In line with relevant studies, we chose a minimum exposition time of $48 \mathrm{~h}$ and an acute follow-up period of 7 days following exposition to detect drug effects independently of other clinical variables which could introduce variance later in the course of burn injury and critical illness. Further, we focused on the established and well-differentiated parameter of serum creatinine per the KDIGO classification to define and detect our endpoints $[35,36]$.

An increase in serum creatinine is a strong indicator of acute kidney damage in adult and pediatric patients and correlates with acute and chronic renal dysfunction in a graded manner [28, 37-39]. Greater elevations predict the highest risk of morbidity and mortality [40, 41]. Even small $\mathrm{Cr} \Delta_{\%} \geq 50 \%$ (which corresponds to KDIGO stage 1) double the probability of adverse outcomes and mortality [42]. Our data showed in both adult and pediatric patients that the relative and absolute creatinine increases following exposition to vancomycin/piperacillin were approximately twice 

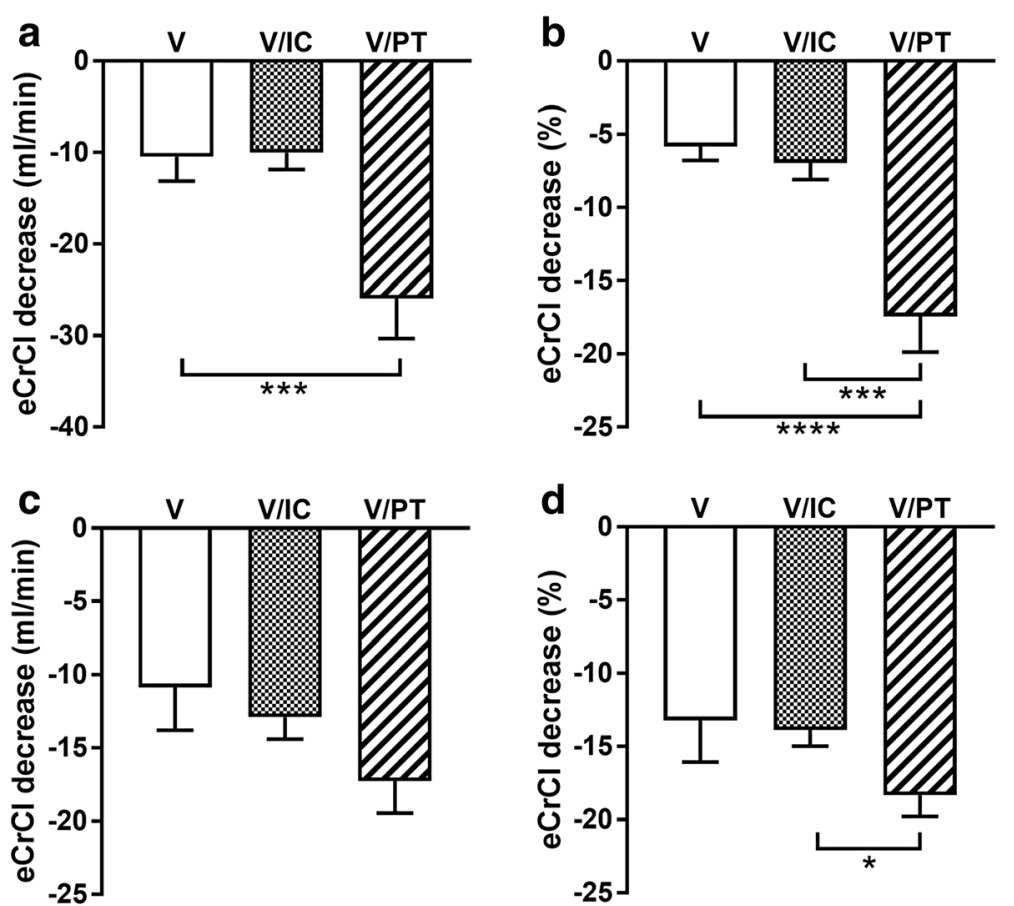

Fig. 3 Adult average (a) absolute (mg/dl) and (b) relative (\%) decrease in eCrCl (calculated via Cockcroft-Gault) 7 days following exposure to V, V/ $I C$, and V/PT. Child average (c) absolute $(\mathrm{mg} / \mathrm{dl})$ and $(\mathbf{d})$ relative (\%) decrease in eCrCl (calculated via Léger's formula) 7 days following exposure to $V, V / I C$, and V/PT. ${ }^{*} p<0.05,{ }^{* *} p<0.001,{ }^{* * * *} p<0.0001$. IC imipenem-cilastin, $P T$ piperacillin-tazobactam, $V$ vancomycin

those observed with vancomycin/imipenem or vancomycin alone. Accordingly, creatinine clearance was reduced after exposure to vancomycin/piperacillin. Clinically, this corresponded to a significantly increased incidence of short-term renal failure in both adults and children, as well as increased renal risk and injury in adults. The observed effects can likely be attributed to the combination of vancomycin/ piperacillin, since the cohorts of $\mathrm{V}$ alone and V/IC behaved similarly to each other, as well as in individual comparison to vancomycin/piperacillin. Interestingly, the lower rates of $\mathrm{AKI}$ in the vancomycin and vancomycin/imipenem groups did not yield hard clinical advantages in terms of length of hospitalization, septicemia, or mortality. While some of these secondary endpoints tended towards statistical significance, the overall sample size may have been insufficient to reliably detect differences.

In our patient cohort, pediatric patients sustained higher TBSA\% burns than adults; therefore, outside of multivariate modeling which reliably corrected for this difference, we analyzed the study groups separately. Our data suggest that the observed effects of antibiotic exposure on the absolute creatinine increase, creatinine clearance decrease,
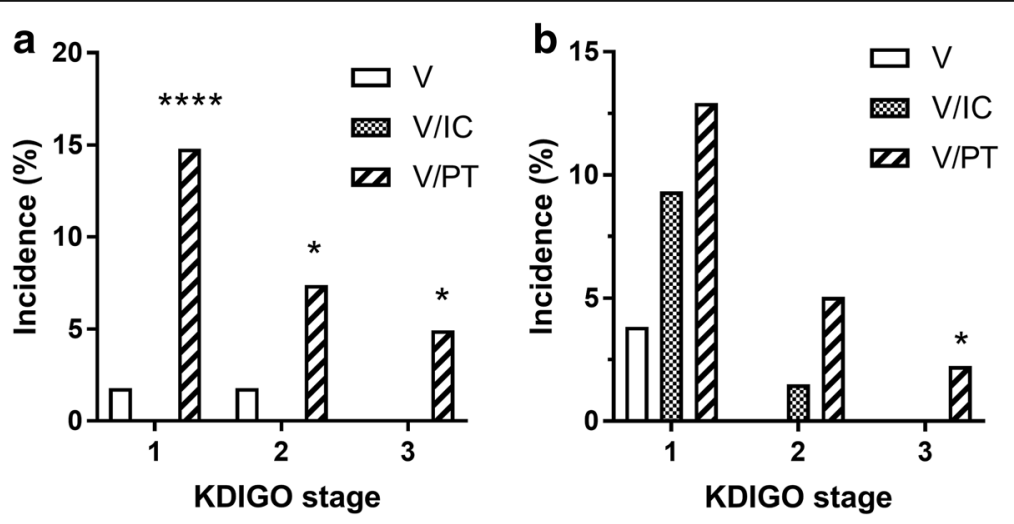

Fig. 4 Relative rate of occurrence (\%) of AKI events according to KDIGO stage 7 days following exposure to V, V/IC, and V/PT in adults (a) and children (b). ${ }^{*} p<0.05,{ }^{* * *} p<0.0001$. IC imipenem-cilastin, KD/GO Kidney Disease Improving Global Outcomes, PT piperacillin-tazobactam, $V$ vancomycin 
Table 2 Clinical characteristics of patients with RRT during hospitalization

\begin{tabular}{|c|c|c|c|c|c|c|c|c|c|c|}
\hline Cohort & TBSA (\%) & LOS (days) & $\mathrm{INH}$ & Septicemia & $\begin{array}{l}\text { First drug } \\
\text { exposure (days) }\end{array}$ & RRT begin (days) & RRT duration (days) & $\mathrm{Cr}_{\mathrm{BL}}(\mathrm{mg} / \mathrm{dl})$ & $\operatorname{Cr} \Delta_{\%}(\%)$ & KDIGO+ \\
\hline \multicolumn{11}{|c|}{ Survivors } \\
\hline V/PT & 62 & 105 & $\mathrm{~N}$ & $\mathrm{~N}$ & 1 & 6 & 76 & 0.8 & 365 & Y \\
\hline V/PT & 80 & 141 & Y & Y & 0 & 40 & 13 & 0.7 & 18 & N \\
\hline V/PT & 32 & 26 & $\mathrm{~N}$ & $\mathrm{~N}$ & 0 & 10 & 2 & 1.01 & 77 & Y \\
\hline \multicolumn{11}{|c|}{ Non-survivors } \\
\hline $\mathrm{V} / \mathrm{IC}$ & 95 & 9 & Y & Y & 1 & 10 & 1 & 0.9 & 0 & $\mathrm{~N}$ \\
\hline V/PT & 62 & 234 & $\mathrm{~N}$ & $\mathrm{~N}$ & 0 & 8 & 18 & 0.9 & 422 & Y \\
\hline V/PT & 45 & 15 & $\mathrm{~N}$ & Y & 1 & 10 & 4 & 0.95 & 21 & $\mathrm{~N}$ \\
\hline V/PT & 40 & 23 & $\mathrm{~N}$ & Y & 0 & 19 & 3 & 0.9 & 30 & $\mathrm{~N}$ \\
\hline
\end{tabular}

$\mathrm{Cr}_{B L}$ baseline creatinine concentration, $\mathrm{Cr} \Delta \%$ relative creatinine increase, IC imipenem-cilastin, INHpresence of inhalation injury at admission, $L O S$ length of hospitalization (days), $N$ no, $P T$ piperacillin-tazobactam,KDIGO+ KDIGO stage 1,2, or 3 during 7 days following exposure, RRT renal replacementtherapy, TBSA total body surface area burned (\%), $V$ vancomycin, $Y$ yes

and the incidence of KDIGO events are greater in the pediatric patient population, while differences between the drug groups appear less pronounced. Several factors may contribute to this effect: children were exposed to higher doses of vancomycin, piperacillin, and imipenem when normalized to body weight, which may have caused increased renal damage in itself; the significantly greater severity of injury and associated systemic critical illness, as well as dilution effects of creatinine due to the more aggressive resuscitation measures taken in this group, may have obscured the differences in creatinine increases. Notwithstanding these potentially confounding variables, the relationship between administration of vancomycin/piperacillin and creatinine increase remains significant and robust, indicating that the observed effect is not exclusive to adults.

With regard to endpoints that surpass the 7-day study window, there was an increased incidence of hemodialysis after antibiotic treatment with vancomycin/piperacillin in the adult patient population. Furthermore, all but one

Table 3 Multivariable linear regression model of dose dependency of continuous outcome creatinine increase

\begin{tabular}{lll}
\hline & \multicolumn{1}{l}{$\mathrm{Cr}_{\%}$} & \\
\cline { 2 - 3 } Coefficients & Estimate & $<$ \\
\hline (Intercept) & 1.5255 & $<0.0001$ \\
[D] PT & 0.0022 & 0.0002 \\
TBSA & 0.0010 & 0.7930 \\
INH & 0.4103 & 0.0379 \\
Age & -0.0057 & 0.1747 \\
[D] V & 0.0007 & 0.8655
\end{tabular}

All logistic models used stepwise variable selection based on minimization of Akaike Information Criterion and passed the Hosmer Lemeshow test for goodness of fit

$C r \Delta_{\%}$ relative creatinine increase (\%), [D] $P T$ average daily dose of piperacillintazobactam (mg/kg/day), TBSA total body surface area burned, INH presence of inhalation injury at admission, [D] $V$ average daily dose of vancomycin coadministered with PT (mg/kg/day) patient who progressed to RRT belonged to the vancomycin/piperacillin cohort. However, these results should be interpreted with caution, as they may have been confounded by a multitude of clinical variables during the time after exposure to vancomycin/piperacillin. This is, in part, supported by the fact that only less than half of subjects who progressed to RRT were identified with a KDIGO-positive event during their initial 7-day study period

The mechanistic explanation of our findings remains elusive; nephrotoxicity of vancomycin is thought to be caused by oxidative stress and acute tubular necrosis [43], which in turn can be promoted by product impurities [15], pre-existing renal dysfunction [44], concomitant critical illness [45], and increased doses and duration of administration [21, 46]. Quite recent experimental data by Luque et al [47]. elucidated the matter further by describing a distinct cast-nephropathy caused by nanospheric intratubular vancomycin aggregates in mice and humans (cit). Most clinical studies do not report incidences of vancomycin-associated acute renal dysfunction of more than $5 \%[45,46]$. Piperacillin-tazobactam in comparison to other $\beta$-lactam antibiotics has been associated with impaired renal recovery by some study groups [48], and an additive detrimental effect on renal function in combination with vancomycin has been proposed [21, 49]. It has been suggested that piperacillin may decrease vancomycin clearance, thus leading to increased accumulation and dosedependent nephrotoxicity of vancomycin [21]. However, this is unlikely to be the case, as comparable doses of vancomycin were administered regardless of the coadministered agent and that administration of vancomycin itself was monitored closely by trough measurements.

Subjects with pre-existing renal conditions were excluded from this study, but it may be concluded that the superimposition of effects of administration of vancomycin/piperacillin on pre-existing acute or chronic 
Table 4 Stepwise logistic regression models of dose dependency of categorical outcomes (KDIGO stages 1,2, and 3)

\begin{tabular}{|c|c|c|c|c|c|c|}
\hline \multirow[b]{3}{*}{ Coefficients } & \multicolumn{6}{|c|}{ KDIGO stage } \\
\hline & \multicolumn{2}{|l|}{1} & \multicolumn{2}{|l|}{2} & \multicolumn{2}{|l|}{3} \\
\hline & Estimate & $p$ & Estimate & $p$ & Estimate & p \\
\hline (Intercept) & -3.9249 & $<0.0001$ & -4.6114 & $<0.0001$ & -4.5883 & $<0.0001$ \\
\hline [D] PT & 0.0036 & 0.0364 & 0.0058 & 0.0039 & 0.0136 & 0.0086 \\
\hline$[D] \vee$ & - & - & - & - & -0.0585 & 0.1057 \\
\hline
\end{tabular}

All logistic models used stepwise variable selection based on minimization of Akaike Information Criterion and passed the Hosmer Lemeshow test for goodness of fit [D] PT average daily dose of piperacillin-tazobactam (mg/kg/day), [D] V average daily dose of vancomycin co-administered with PT (mg/kg/day), KDIGO Kidney Disease Improving Global Outcomes

kidney injury should be avoided, especially in this at-risk population. Furthermore, the low incidence of KDIGO events in association with vancomycin alone confirms other recent reports $[43,50]$ which estimate the nephrotoxicity of vancomycin to be lower than commonly described in older literature [51]. Clearly, further research, perhaps in a reliable animal model which allows for analysis of morphologic renal effects, is warranted to elucidate this phenomenon further.

The clinical implications of this study may well extend beyond pediatric and adult burn care. Given the increasing body of evidence suggesting that even small increases in creatinine are indicative of substantially worse outcomes of morbidity and mortality, every effort should be made to reduce nephrotoxicity. Our data suggest that combination therapy of imipenem-cilastin with vancomycin may be advantageous over piperacillin-tazobactam in burn patients, which led to a change in our centers' clinical practice guidelines in light of the emerging findings of others and this study. However, it needs to be strongly emphasized that replacing piperacillin with imipenem bears great risks in itself, outside potential benefits regarding nephrotoxicity. The increased use of carbapenems has been linked to a substantial increase in multidrug-resistant $P$. aeruginosa, A. baumanii, or $S$. maltophilia in various clinical settings and is certainly a concern in burn care [52-54]. Potential ways to mitigate both risks might be to combine vancomycin with other antibiotics with lower resistance-inducing potential or to seek alternatives to high-dose systemic therapy with vancomycin altogether to reduce nephrotoxic potential.

There are several limitations to this study that warrant consideration. The single center, retrospective design precludes inferences which could have been made from a prospectively designed randomized trial. This study focuses on the serum creatinine definition of KDIGO classification as diuresis data was not available in a sufficiently comprehensive manner to be included into the analysis; however, this affects all treatment and age groups equally and should therefore not skew the analysis, despite a tendency to potentially underestimate the incidence of AKI. Furthermore, sensitivity of serum creatinine alone has been reported to be sufficient in detecting AKI and to be more precise than urinary output measures. This study does not have a control group of piperacillin monotherapy to allow for inferences towards whether the observed toxicity of vancomycin/piperacillin is attributable to vancomycin, piperacillin, or both. In light of the large body of evidence that suggests no individual toxicity of piperacillin, future studies will need to verify the exact mechanism underlying the observed phenomenon.

Adjustment for injury severity in this study was based on age, TBSA burned, and the presence of inhalation injury, as these are the most potent predictors in acute burn injury. The lack of established scores such as IGS2 or SOFA as adjustors limits this study's comparability with other critical care patient collectives outside of burn care.

The group of pediatric patients who received vancomycin alone is disproportionately smaller than the other cohorts. As the children in this trial were more severely burned and had higher incidence of inhalation injury, antibiotic monotherapy was less likely from a clinical standpoint. Furthermore, evaluation of other potentially confounding nephrotoxic agents (such as iodinated contrast agents, aminoglycosides, vasopressors, etc.) administered during the 7 days of monitoring was outside the scope of this study. Because of the high degree of standardization in all aspects of burn injury treatment at our centers, we can assume that potential confounders should affect all treatment cohorts equally and cannot account for observed effects such as the significantly greater creatinine increase averaged over all study patients. The bacteriological indication and efficacy of the antibiotics administered in this study have been described elsewhere $[11,55-58]$ and were not evaluated to maintain focus on their side effects. Lastly, patients were treated earlier in time with the combination of vancomycin/piperacillin because we discontinued this combination in favor of vancomycin/imipenem when preliminary data became available regarding its potential detrimental effects. While this could in theory skew the analysis towards more positive outcomes due to improvements in care in more recent years, treatment and overall outcomes (such as gross mortality) at our centers have not significantly 
changed in the median 4 years of time difference between the groups, which makes a confounding effect unlikely. To the contrary, this unintentional "before-andafter" design of this retrospective analysis strengthens its results by equalizing the propensity to receive either V/PT (before) or $\mathrm{V} / \mathrm{IC}$ (after) for all patients over time and thus preventing potential selection bias.

\section{Conclusions}

The co-administration of vancomycin and piperacillintazobactam is associated with disproportionally elevated serum creatinine and lower creatinine clearance when compared to vancomycin alone or its combination with imipenem-cilastin. This increase in serum creatinine is indicative of increased incidence of renal dysfunction based on the KDIGO criteria. The tradeoff between potentially decreased nephrotoxicity of vancomycin/impinenem-cilastin and the risk of induction of multidrug-resistant organisms due to the potential overuse of carbapenems remains a clinical challenge.

\section{Additional file}

Additional file 1: Table S1. Statistical summary of secondary study endpoints. All data are presented as count (percentage) or mean (standard deviation). (DOCX $12 \mathrm{~kb}$ )

\section{Abbreviations}

AKl: Acute kidney injury; ANOVA: Analysis of variance; Cr: Creatinine; eCrCl: Estimated creatinine clearance; IC: Imipenem-cilastin; KDIGO: Kidney Disease Improving Global Outcomes; PT: Piperacillin-tazobactam; RRT: Renal replacement therapy; TBSA: Total body surface area; UTMB: University of Texas Medical Branch; V: Vancomycin

\section{Acknowledgements}

None.

\section{Funding}

This work was supported by NIH (P50 GM060338, R01 GM056687, R01 HD049471, R01 GM112936, R01-GM56687, and T32 GM008256), Shriners Hospitals for Children (84080, 80100, 71008, and 71000), and was in part supported by a Clinical and Translational Science Award (UL1TR000071) from the National Center for Advancing Translational Sciences $(\mathrm{NIH})$. None of the funding sources had any role in the design of the study, in the collection, analysis, or interpretation of the data, or in the writing of the manuscript.

\section{Availability of data and materials}

The datasets used and/or analyzed during the current study are available from the corresponding author on reasonable request.

\section{Authors' contributions}

All authors contributed substantially in the conception of this manuscript. In detail, GH collected and structured data, performed statistical analyses, and drafted the manuscript. DNH, CCF, LKB, and WBN conceived of the study, and participated in its design and coordination. KDC performed the linear and logistic data modeling and advised on statistical matters. MS, ADC, EAK, $J C D$, and CDV assisted in data collection and structuring of data, as well as database management; they also revised the manuscript. RPM and MPK provided major assistance in the interpretation of clinical data in relation to this manuscript's hypotheses and endpoint-related questions. DNH, CCF, WBN, and MPK helped to draft the manuscript and revised it. All authors read and approved the final manuscript.

\section{Ethics approval and consent to participate}

This retrospective chart and database review was approved by the University of Texas Medical Branch Institutional Review Board and the Shriners Hospitals for Children (SHC) Office for Clinical Research. No additional patient consent was needed for this retrospective review per the UTMB and SHC Institutional Review Boards.

\section{Consent for publication}

Not applicable.

\section{Competing interests}

The authors declare that they have no competing interests.

\section{Author details}

'Department of Surgery, University of Texas Medical Branch, 301 University Blvd., Galveston, TX 77555, USA. ${ }^{2}$ Shriners Hospitals for Children, 815 Market St., Galveston, TX 77550, USA. ${ }^{3}$ Department of Hand, Plastic and Reconstructive Surgery, Burn Trauma Center, BG Trauma Center Ludwigshafen, University of Heidelberg, Ludwig-Guttmann-Str. 13, 67071 Ludwigshafen, Germany. ${ }^{4}$ Department of Plastic Surgery, Baylor College of Medicine, 1 Baylor Plaza, Houston, TX 77030, USA. ${ }^{5}$ Division of Plastic, Aesthetic and Reconstructive Surgery, Department of Surgery, Medical University of Graz, Graz, Austria. ${ }^{6}$ Department of Anesthesiology, University of Texas Medical Branch, 301 University Blvd., Galveston, TX 77555, USA.

Received: 8 August 2017 Accepted: 28 November 2017

Published online: 20 December 2017

References

1. Holm C, Hörbrand F, von Donnersmarck GH, Mühlbauer W. Acute renal failure in severely burned patients. Burns. 1999;25(2):171-8.

2. Chrysopoulo MT, Jeschke MG, Dziewulski P, Barrow RE, Herndon DN. Acute renal dysfunction in severely burned adults. J Trauma Acute Care Surg. 1999;46(1):141-4.

3. Uchino S, Kellum JA, Bellomo R, Doig GS, Morimatsu H, Morgera S, et al. Acute renal failure in critically ill patients: a multinational, multicenter study. Jama. 2005:294(7):813-8.

4. Jeschke MG, Barrow RE, Wolf SE, Herndon DN. Mortality in burned children with acute renal failure. Arch Surg. 1998;133(7):752-6.

5. Steinvall I, Bak Z, Sjoberg F. Acute kidney injury is common, parallels organ dysfunction or failure, and carries appreciable mortality in patients with major burns: a prospective exploratory cohort study. Crit Care. 2008;12:R124.

6. Klevens RM, Edwards JR, Tenover FC, McDonald LC, Horan T, Gaynes R. Changes in the epidemiology of methicillin-resistant Staphylococcus aureus in intensive care units in US hospitals, 1992-2003. Clin Infect Dis. 2006;42(3): 389-91.

7. Weinstein RA, Gaynes R, Edwards JR. Overview of nosocomial infections caused by gram-negative bacilli. Clin Infect Dis. 2005:41(6):848-54.

8. Siegel JD, Rhinehart E, Jackson M, Chiarello L. Management of multidrugresistant organisms in health care settings, 2006. Am J Infect Control. 2007; 35(10):S165-93.

9. Stanojcic $M$, Chen $P$, Xiu F, Jeschke MG. Impaired immune response in elderly burn patients: new insights into the immune-senescence phenotype Ann Surg. 2016;264(1):195-202.

10. Jeschke MG, Patsouris D, Stanojcic M, Abdullahi A, Rehou S, Pinto R, et al. Pathophysiologic response to burns in the elderly. EBioMedicine. 2015;2(10): 1536-48.

11. Church D, Elsayed S, Reid O, Winston B, Lindsay R. Burn wound infections. Clin Microbiol Rev. 2006:19(2):403-34

12. Weber JM, Sheridan RL, Pasternack MS, Tompkins RG. Nosocomial infections in pediatric patients with burns. Am J Infect Control. 1997;25(3):195-201.

13. Williams FN, Herndon DN, Hawkins HK, Lee JO, Cox RA, Kulp GA, et al. The leading causes of death after burn injury in a single pediatric burn center. Crit Care. 2009;13(6):1.

14. Liu C, Bayer A, Cosgrove SE, Daum RS, Fridkin SK, Gorwitz RJ, et al. Clinical practice guidelines by the Infectious Diseases Society of America for the treatment of methicillin-resistant Staphylococcus aureus infections in adults and children. Clin Infect Dis. 2011:52(3):e18-e55.

15. Elyasi S, Khalili H, Dashti-Khavidaki S, Mohammadpour A. Vancomycininduced nephrotoxicity: mechanism, incidence, risk factors and special populations. A literature review. Eur J Clin Pharmacol. 2012;68(9):1243-55. 
16. Buckley MM, Brogden RN, Barradell LB, Goa KL. Imipenem/cilastatin. Drugs. 1992:44(3):408-44.

17. Bourget $\mathrm{P}$, Lesne-Hulin A, Reveillé RL, Bever HL, Carsin H. Clinical pharmacokinetics of piperacillin-tazobactam combination in patients with major burns and signs of infection. Antimicrob Agents Chemother. 1996; 40(1):139-45.

18. Brismar B, Malmborg AS, Tunevall G, Wretlind B, Bergman L, Mentzing LO, et al. Piperacillin-tazobactam versus imipenem-cilastatin for treatment of intraabdominal infections. Antimicrob Agents Chemother. 1992;36(12):2766-73.

19. Erasmo AA, Crisostomo AC, Yan L-N, Hong Y-S, Lee K-U, Lo C-M. Randomized comparison of piperacillin/tazobactam versus imipenem/ cilastatin in the treatment of patients with intra-abdominal infection. Asian J Surg. 2004;27(3):227-35.

20. Joly-Guillou M-L, Kempf M, Cavallo J-D, Chomarat M, Dubreuil L, Maugein J, et al. Comparative in vitro activity of meropenem, imipenem and piperacillin/tazobactam against 1071 clinical isolates using 2 different methods: a French multicentre study. BMC Infect Dis. 2010;10:72.

21. Burgess $L D$, Drew RH. Comparison of the incidence of vancomycin-induced nephrotoxicity in hospitalized patients with and without concomitant piperacillin-tazobactam. Pharmacother J Hum Pharmacol Drug Ther. 2014; 34(7):670-6.

22. Gomes DM, Smotherman C, Birch A, Dupree L, Della Vecchia BJ, Kraemer DF, et al. Comparison of acute kidney injury during treatment with vancomycin in combination with piperacillin-tazobactam or cefepime. Pharmacother J Hum Pharmacol Drug Ther. 2014;34(7):662-9.

23. Lameire NH, Bagga A, Cruz D, De Maeseneer J, Endre Z, Kellum JA, et al. Acute kidney injury: an increasing global concern. Lancet. 2013;382(9887):170-9.

24. Ali A, Herndon DN, Mamachen A, Hasan S, Andersen CR, Grogans R-J, et al. Propranolol attenuates hemorrhage and accelerates wound healing in severely burned adults. Crit. Care Lond. Engl. 2015;19:217.

25. Baxter CR, Shires T. Physiological response to crystalloid resuscitation of severe burns. Ann N Y Acad Sci. 1968;150(1):874-94.

26. Jeschke MG, Gauglitz GG, Finnerty CC, Kraft R, Mlcak RP, Herndon DN. Survivors versus non-survivors postburn: differences in inflammatory and hypermetabolic trajectories. Ann Surg. 2014;259(4):814-23.

27. Jeschke MG, Chinkes DL, Finnerty CC, Kulp G, Suman OE, Norbury WB, et al. Pathophysiologic response to severe burn injury. Ann Surg. 2008;248(3):387-401.

28. Bagshaw SM, George C, Bellomo R. A comparison of the RIFLE and AKIN criteria for acute kidney injury in critically ill patients. Nephrol Dial Transplant. 2008;23(5):1569-74.

29. Cockcroft DW, Gault H. Prediction of creatinine clearance from serum creatinine. Nephron. 1976;16(1):31-41.

30. Léger F, Bouissou F, Coulais Y, Tafani M, Chatelut E. Estimation of glomerular filtration rate in children. Pediatr Nephrol Berl Ger. 2002;17(11):903-7.

31. Posada D, Buckley TR. Model selection and model averaging in phylogenetics: advantages of akaike information criterion and Bayesian approaches over likelihood ratio tests. Syst Biol. 2004;53(5):793-808.

32. Hosmer DW, Hosmer T, Le Cessie S, Lemeshow S. A comparison of goodness-of-fit tests for the logistic regression model. Stat Med. 1997; 16(9):965-80.

33. Moenster RP, Linneman TW, Finnegan PM, Hand S, Thomas Z, McDonald JR. Acute renal failure associated with vancomycin and $\beta$-lactams for the treatment of osteomyelitis in diabetics: piperacillin-tazobactam as compared with cefepime. Clin Microbiol Infect. 2014;20(6):O384-9.

34. Hellwig T, Hammerquist R, Loecker B, Shields J. 301: retrospective evaluation of the incidence of vancomycin and/or piperacillin-tazobactam induced acute renal failure. Crit Care Med. 2011;39(12):79.

35. Palevsky PM, Liu KD, Brophy PD, Chawla LS, Parikh CR, Thakar CV, et al. KDOQI US commentary on the $2012 \mathrm{KDIGO}$ clinical practice guideline for acute kidney injury. Am J Kidney Dis. 2013;61(5):649-72.

36. Roy AK, Mc Gorrian C, Treacy C, Kavanaugh E, Brennan A, Mahon NG, et al. A comparison of traditional and novel definitions (RIFLE, AKIN, and KDIGO) of acute kidney injury for the prediction of outcomes in acute decompensated heart failure. Cardiorenal Med. 2013;3(1):26-37.

37. Akcan-Arikan A, Zappitelli M, Loftis LL, Washburn KK, Jefferson LS, Goldstein SL. Modified RIFLE criteria in critically ill children with acute kidney injury. Kidney Int. 2007;71(10):1028-35.

38. Waikar SS, Bonventre JV. Creatinine kinetics and the definition of acute kidney injury. J Am Soc Nephrol. 2009;20(3):672-9.
39. Palmieri T, Lavrentieva A, Greenhalgh D. An assessment of acute kidney injury with modified RIFLE criteria in pediatric patients with severe burns. Intensive Care Med. 2009;35(12):2125.

40. Smith GL, Vaccarino V, Kosiborod M, Lichtman JH, Cheng S, Watnick SG, et al. Worsening renal function: what is a clinically meaningful change in creatinine during hospitalization with heart failure? J Card Fail. 2003;9(1):13-25.

41. Ishani A, Nelson D, Clothier B, Schult T, Nugent S, Greer N, et al. The magnitude of acute serum creatinine increase after cardiac surgery and the risk of chronic kidney disease, progression of kidney disease, and death. Arch Intern Med. 2011;171(3):226-33.

42. Lassnigg A, Schmid ER, Hiesmayr M, Falk C, Druml W, Bauer P, et al. Impact of minimal increases in serum creatinine on outcome in patients after cardiothoracic surgery: do we have to revise current definitions of acute renal failure? Crit Care Med. 2008;36(4):1129-37.

43. Gupta A, Biyani M, Khaira A. Vancomycin nephrotoxicity: myths and facts. Neth J Med. 2011;69(9):379-83.

44. Lodise TP, Lomaestro B, Graves J, Drusano GL. Larger vancomycin doses (at least four grams per day) are associated with an increased incidence of nephrotoxicity. Antimicrob Agents Chemother. 2008;52(4):1330-6.

45. Jeffres MN, Isakow W, Doherty JA, Micek ST, Kollef MH. A retrospective analysis of possible renal toxicity associated with vancomycin in patients with health care-associated methicillin-resistant Staphylococcus aureus pneumonia. Clin Ther. 2007;29(6):1107-15.

46. Van Hal SJ, Paterson DL, Lodise TP. Systematic review and meta-analysis of vancomycin-induced nephrotoxicity associated with dosing schedules that maintain troughs between 15 and 20 milligrams per liter. Antimicrob Agents Chemother. 2013;57(2):734-44.

47. Luque Y, Louis K, Jouanneau C, Placier S, Esteve E, Bazin D, et al. Vancomycin-Associated Cast Nephropathy. J Am Soc Nephrol. 2017;28: 1723-8.

48. Jensen J-US, Hein L, Lundgren B, Bestle MH, Mohr T, Andersen MH, et al. Kidney failure related to broad-spectrum antibiotics in critically ill patients: secondary end point results from a 1200 patient randomised trial. BMJ Open. 2012;2(2):e000635.

49. Min E, Box K, Lane J, Sanchez J, Coimbra R, Doucet J, et al. 714: acute kidney injury in patients receiving concomitant vancomycin and piperacillin/tazobactam. Crit Care Med. 2011;39(12):200.

50. Hidayat LK, Hsu DI, Quist R, Shriner KA, Wong-Beringer A. High-dose vancomycin therapy for methicillin-resistant Staphylococcus aureus infections: efficacy and toxicity. Arch Intern Med. 2006;166(19):2138-44.

51. Bailie GR, Neal D. Vancomycin ototoxicity and nephrotoxicity. Med Toxicol Adverse Drug Exp. 1988;3(5):376-86.

52. Troillet N, Samore MH, Carmeli Y. Imipenem-resistant pseudomonas aeruginosa: risk factors and antibiotic susceptibility patterns. Clin Infect Dis. 1997;25(5):1094-8

53. Pournaras S, Markogiannakis A, Ikonomidis A, Kondyli L, Bethimouti K, Maniatis AN, et al. Outbreak of multiple clones of imipenem-resistant acinetobacter baumannii isolates expressing OXA-58 carbapenemase in an intensive care unit. J Antimicrob Chemother. 2006;57(3):557-61.

54. Keen EF, Robinson BJ, Hospenthal DR, Aldous WK, Wolf SE, Chung KK, et al. Prevalence of multidrug-resistant organisms recovered at a military burn center. Burns. 2010;36(6):819-25.

55. Moncrief JA, Switzer WE, Order SE, Mills Jr W, Lindberg RB. The successful control of burn wound sepsis. J Trauma Acute Care Surg. 1965;5(5):601-16

56. Sheridan RL. Sepsis in pediatric burn patients. Pediatr Crit Care Med. 2005: 6(3):S112-9.

57. Pruitt Jr BA, McManus AT, Kim SH, Goodwin CW. Burn wound infections: current status. World J Surg. 1998;22(2):135-45.

58. Dacso CC, Luterman A, Curreri PW. Systemic antibiotic treatment in burned patients. Surg Clin North Am. 1987;67(1):57-68. 